La mirada de Adolf Loos sobre el café vienés como tercer lugar Jesús López Baeza

páginas / año 12 - n 29 Mayo-Agosto / ISSN 1851-992X/ 2020

http://revistapaginas.unr.edu.ar/index.php/RevPaginas

\title{
La mirada de Adolf Loos sobre el café vienés como tercer lugar
}

\author{
Adolf Loos' approach to the Viennese coffee house as a "third place"
}

\begin{abstract}
Resumen
Durante el período correspondiente al cambio del siglo XIX al XX en Viena, se persigue un estilo propio y único, der Wiener Stil, que diferencie al imperio Habsburgo de Alemania, del resto de Europa, y de la tradición historicis-ta. Esta búsqueda de una identidad propia se aborda desde varios prismas importantes o corrientes estilísticas, con preocupaciones e intenciones diferentes y algunos puntos en común. El pensamiento propio de cada corriente se refleja en su estilo formal y es resultado de los procesos de gestación y generación de las mismas. Asimismo, la figura del café vienés es fundamental, en términos sociales y culturales, para comprender estos procesos. En este contexto, resulta imprescindible el trabajo del arquitecto Adolf Loos que ha sido investigado en múltiples ocasiones y desde diversas disciplinas, al igual que su concepción sobre la publicidad y la privacidad; sin embargo, cómo éstas afectan a los espacios que se sitúan en el límite entre lo público y lo privado, todavía no ha sido tratado en profundidad. Este trabajo analiza el espacio del café vienés como un lugar en el que el carácter de lo público y de lo doméstico intersectan y se profundiza en ellos desde el prisma de pensamiento loosiano, en relación con las co-rrientes coetáneas.
\end{abstract}

Palabras clave

Viena; Café; Adolf Loos; Tercer Lugar

Esta obra está sujeta a la Licencia Reconocimiento-NoComercial-CompartirIgual 4.0 Internacional de Creative Commons. http://creativecommons.org/licenses/by-nc-sa/4.0/ (cc) BY-NC-SA 


\begin{abstract}
During the turn of the 19th century in Vienna, a novel and unique artistic style was pursued. In German, "der Wiener Stil" was intended to differentiate the Habsburg Empire's style from the previous historicist tradition, and from the aesthetics trends followed by the rest of European countries. This search for an own identity is approached from several important perspectives and performed through a set of stylistic trends, all of them having different concerns and intentions, and some points in common. The thoughts of each artistic trend are reflected on its formal style and they are the result of specific processes of generation. In this con-text, the role of Viennese coffee house is fundamental to understand these processes both in social and cultural terms. Within this frame of reference, the work of the architect Adolf Loos has been a popular research topic for several disciplines, with special focus on his essential conception of publicity and privacy; however, the way this conception affects space - and especially when on the intersection between the public and the private realms - has not yet been discussed in depth. Thus, the present work analyzes the figure of the Viennese café as a place where the public and the domestic intersect. It does so through the prism of Loos' thought and in relation to Austrian artistic trends.
\end{abstract}

\title{
Keywords
}

Vienna; Café; Adolf Loos; Third 


\section{Un preludio}

La riqueza y complejidad sociocultural en la transición del siglo XIX al XX se muestra evidente. Responde a unas tendencias sin precedentes, influidas por nuevos descubrimientos tecno-científicos que sitúan a la ciencia como motor de desarrollo del conocimiento y la cultura. Los tiempos se perciben como diferentes, más modernos e independientes del contexto histórico hasta ese momento. Es por ello que los estilos artísticos manifiestan una voluntad de ruptura con la estética precedente. La ciudad de Viena es el centro económico y cultural de Europa central y la sede de muchas de estas nuevas ideas y conceptos que aspiran a renovar y redefinir el pensamiento científico y los estilos artísticos por completo (Franz 2013). La Viena del fin-de-siècle ${ }^{1}$ no es únicamente la capital administrativa del imperio Habsburgo, sino que también constituye la principal base económica y cultural en la que éste se sustenta, instituyéndose como un corazón creativo en el que se establecerían artistas, hombres de ciencia y arquitectos como Adolf Loos, Joseph Hoffmann, Sigmund Freud o Gustav Klimt (Franz 2013).

En este contexto, el estudio del pensamiento del arquitecto Adolf Loos es fundamental para la comprensión de la reflexión arquitectónica en las narrativas actuales. Como reza el título de la exposición de Beatriz Colomina de 2013 en GSAPP, Loos es "nuestro contemporáneo" (Colomina 2013)². Si bien es cierto que el personaje de Adolf Loos ha sido investigado en múltiples ocasiones y desde diversos puntos de vista, su concepción de la publicidad y la privacidad, y cómo éstas afectan a los espacios que se sitúan en la intersección entre lo público y lo privado - los terceros lugares ${ }^{3}$-, resulta de interés para completar una visión contemporánea sobre el arquitecto austriaco en su contexto del fenómeno renovador global.

Este planteamiento pone en relación con la herencia histórica inmediatamente anterior al momento del cambio de siglo XIX al XX en Viena, con el nacimiento de los diferentes estilos de la modernidad ${ }^{4}$, sus manifiestos o ideales, y el reflejo de los mismos en sus características formales definitorias. Posteriormente, se evalúa la

\footnotetext{
1 Fin-de-siècle: Se utiliza el término en francés original, ya que así se encuentra presente en textos de referencia tanto en inglés como alemán, refiriéndose al período de los últimos años del siglo XIX y los primeros del XX.

2 Texto para la exposición Adolf Loos: Our Contemporaryen el Arthur Ross Architecture Gallery, Buell Hall de la Graduate School of Architecture, Planning and Preservation de la Universidad de Columbia. 2013.

${ }^{3}$ El término "Terceros Lugares" es tomado según la definición de Third Places de Oldenburg (1989) como lugares de interacción y concentración de capital social, que por definición - "A home away from home"- contienen carácter doméstico, y a la vez público.

4 Modernidad, Modernismo: Se han tomado ambas palabras como sinónimos. Si bien es cierto que existen matices, las ediciones traducidas que se han consultado las utilizan como equivalentes desde el término inglés modernism, referido al movimiento caracterizado por cambios filosóficos, sociológicos, artísticos, culturales, científicos, y arquitectónicos que transforman la sociedad en los últimos años del siglo XIX y los primeros del XX.
} 
importancia del café 5 como foro cultural de intercambio de ideas y discusiones intelectuales, y su papel en este contexto a través del prisma de pensamiento de Adolf Loos como precursor de muchos de los cambios mencionados.

\section{El estilo de la Viena de 1900}

La cultura europea de fin-de-Siècle se caracteriza por una enorme cantidad de estilos artísticos que preceden o dan lugar a la época contemporánea. Estos son fruto de una gran diversidad de corrientes de pensamiento influidas por puntos de vista en ocasiones complementarios, y en ocasiones contrapuestos. Menz (2011) describe este periodo, para la ciudad de Viena, como "el momento en que un grupo de jóvenes artistas, arquitectos, escritores, músicos, diseñadores y pensadores transgredieron las normas para crear un nuevo mundo". Afirma también que "esta revolución artística transformó Viena en una metrópolis dinámica a la vanguardia del modernismo más innovador" ${ }^{\prime}$

Esta nueva era de modernidad no se limita únicamente a las artes plásticas o la arquitectura. Elegancia, osadía y opulencia se combinaban para dar lugar a una nueva etapa de pensamiento y cultura. Comenzaba a explorarse la sexualidad, la psicología, los sueños, el erotismo, tanto desde el arte como desde la más pura ciencia. En este contexto, el límite entre lo público y lo privado se desdibuja (Colomina 1992), y la moralidad queda cuestionada (Schwartz 2012; Szeps and Szeps-Zuckerkadl 1938).

En el campo de la arquitectura y las artes decorativas, es el estilo la cuestión que más polémica desata (Reynolds 1997; Varnedoe 1986). A mediados del siglo XIX, los estilos inspirados en la historia eran los que recibían mayor impulso, sirviendo de inspiración para nuevos diseños tanto en la arquitectura como en los pequeños objetos (Reynolds 1997; Loos 2012b). Este historicismo se basaba principalmente en diseños orientales, y diseños de estilo renacentista centroeuropeo. Este fenómeno conduce a que en la década de 1890, un estilo propio del siglo XIX no hubiera aparecido todavía. En Alemania, el estilo renacentista alemán se veía como un modo de representar la unidad nacional bajo un nuevolenguaje de diseño, según lo defendían teóricos y críticos como Georg Hirth o Julius Lessing (Reynolds 1997), para los que la nueva Alemania debía, ante todo, tener una identidad estética propia para conseguir una unidad patriótica. El revival del Renacimiento alemán se convirtió en la estética del Reich.

Esta mirada al pasado quizás demasiado profunda neutraliza el arte impidiendo que éste se desarrolle de manera natural (Riegel 1895)7 ${ }^{7}$ Consecuentemente teóricos

\footnotetext{
${ }^{5}$ Café: Se utiliza como equivalente los términos en inglés cafe, coffeehouse y en alemán Kaffeehaus. No debe confundirse con el término inglés cafeteria la cual se caracteriza por los mostradores de comida rápida y self service presentes en comedores comunes, o con la bebida caliente coffee.

${ }^{6}$ Texto para la exposición Melbourne Winter Masterpieces 2011 en el National Galery of Victoria International.

7 También citado por Reynolds Geschichte Des Deutschen Kunstgewerbes (Berlin: G. Grote, 1888).
} 


\section{La mirada de Adolf Loos sobre el café vienés como tercer lugar}

como Von Falke (1888) o Von Eitelberger (1879) critican este fenómeno en el que estilos revivalno representarían la situación cultural del momento, sino que habrían sido impuestos deliberadamente con intenciones políticas. Es por ello que la decisión que se toma en Austria conduce a la emancipación del historicismo para inclinarse por diseños ligados a justificaciones intelectuales (Folnesics 1890; WittDörring 2013a). Muthesius (1908) lo describe como un acercamiento desinteresado a la ciencia que, lejos de tratarse de la búsqueda de una identidad en el pasado romántico y emocional al estilo de los alemanes, supondría un nuevo estilo propio que reflejaría el espíritu científico de la nueva era.

Es aquí cuando las artes decorativas surgen como un modo de renovación de la cultura, elevándose a una categoría superior al reconocerse su capacidad de influir o determinar los modos en los que las personas viven, se comportan, y se relacionan (Muthesius 1908) ${ }^{8}$. Evidenciando la mutua relación entre arte y ciencia y los nuevos modos de pensar y en particular, la sustancial influencia de la psicología conductista, Protig (1883) enuncia: "Zeig mir, was du um dich und an dir hast, und ich will dir sagen, wer du bist", dime lo que llevas puesto y lo que tienes alrededor', y te diré quién eres. La ciencia y los estilos de diseño se influyen entre si, hacen preguntas que el otro responde, y se sirven de mutua fuente de inspiración.

En Viena, el desarrollo del arte en la época moderna queda, al principio, postergado por cuestiones económicas, y enfocado al beneficio de los artistas. Jensen (1996) afirma que el nacimiento de la Sezession (1897-1905) supone la evolución de un modelo de negocio basado en el mecenas, a otro nuevo modelo orientado en el sistema comercial de compradores y productores privados. Dicho colectivo surge como un grupo novedoso de arquitectos, diseñadores gráficos y artistas decorativos, con una nueva y variada estética diametralmente opuesta a los estilos revival previos (Fiell and Fiell 1999). Nace de un pequeño grupo de artistas que se reúne por primera vez como el Siebener Klub en el café Sperl de Viena (Fahr-Becker 2008). Su lema "Der Kunst ihre Zeit, der Zeit ihre Freiheit", a cada tiempo su arte, y a cada arte su libertad ${ }^{10}$, hace referencia a este establecimiento de un estilo nuevo y propio para el momento, y al mismo tiempo a la ruptura con la tradición artista-mecenas. El arte es algo vivo y libre, que no debe responder a una intención política o comercial (Bahr 1899).

Este estilo está basado en tres pilares principales: los trabajos de Koloman Moser y sus influencias japonesas, en el Flächenkunst, las influencias clasicistas e historicistas de las décadas previas, la conocida como era Bidermeier, y por último, las artes populares (Witt-Dörring 2013b). El estilo de la Sezession varía por cada uno de sus autores, pero las líneas generales apuntan hacia la geometrización, las

\footnotetext{
8 "[...] die Kunstgewerbe ist zu einer Kulturbewegung im weitesten Sinne des Wortes geworden,die unsere Sitte des Wohnens, des Umganges, der Geselligkeit beeinflufit." También citado por Hess Marketing Modernism in Fin-de-Siècle Europe (Princeton University Press, 1996).

${ }^{9}$ En alemán original, traducido por el autor

${ }^{10}$ En alemán original, traducido por el autor
} 
líneas rectas, las fuertes simetrías, contrastes entre materiales y texturas, y el uso de acabados metálicos o dorados.

En cuanto a su adscripción conceptual, el movimiento de Sezession vienesa se asocia con el pensamiento riegeliano del diseño con voluntad específica de convertirse en arte. Con su fundación, lo que se pretende es integrar el arte en el diseño de los objetos de la vida diaria, con un estilo propio y definitorio que los dote de identidad. Así, mejorando la calidad de los bienes a través de un mayor cuidado de la estética se conseguiría una mejora de la calidad de vida de las personas. Ludwig Hervesi describe su obra como mágica (Fahr-Becker 2008; Lajos Hevesi 1906). Otto Wagner, en contraste al ideal de la popularización de los estilos revival en Alemania en las décadas anteriores, define el estilo sezessionista como un Nacimiento frente al renacimiento del Renacimiento (Fahr-Becker 2008).

En 1897 la Sezession se funda con uno de sus principales propósitos de crear un estilo austríaco inimitable, único y propio, pero que, con la perspectiva de la historia, acaecida décadas después, acaba convirtiéndose en el estilo vienés o Wiener Stil. Sin embargo, la creciente y rápida popularidad de Sezession se convertiría la principal amenaza del principal activo de Sezession, ser diferentes en estilo. Klimt, Hoffmann y Moser se convierten en la última moda, "con toda la tiranía sin sentido que la moda tiene" (Bahr 1899)11. En este momento, el espacio social sufre grandes transformaciones. Desde lecturas públicas a discusiones de café, la opinión pública tiene un papel extraordinario en las narrativas la ciudad (Stewart 2013, 2009). La crítica y lo que el público opina determina lo que interesa, lo que es importante, y en última instancia, lo que lo define. Bahr (1899) distingue dos tipos de crítica, en la Viena de 1900. Por un lado, la antigua crítica, que influye directamente en el arte en términos comerciales, sirve de guía al comprador y, consecuentemente, influye en la producción de arte. Por otro lado, la nueva crítica que comienza a desarrollarse en este momento y analiza las obras posicionándose en el punto de vista psicológico del artista mismo (Bahr 1899). Es por esto que con la evolución de la Sezession hacia un estado de popularidad, ésta comienza a ser considerada comercialy la crítica se torna negativa (Hess 2006).

Seguidamente y como consecuencia de este proceso, el movimiento de la Wiener Werkstätte se convierte en uno de los beneficiarios de este fenómeno. En este contexto, es necesario destacar la figura de Berta Zuckerkandl, que junto con Ludwig Hervesi lanzan a la Wiener Werkstätte por primera vez a las páginas de FremdenBlatt en 1905. La Wiener Werkstätte se funda en 1903 por los sezessionistas Josef Hoffmann y Koloman Moser con una gran influencia de las organizaciones de Arts and Crafts inglesas que defienden el arte a través de las artes decorativas y la artesanía (Fiell and Fiell 1999) (Fig. 1). Fue su experiencia como profesores en la Wiener Kunstgewerbeschule junto con Alfred Roller, lo que les permite transmitir e

\footnotetext{
11 "Hat noch vor einem Jahr fast Courage dazu gehört, für die Sezession, so gehört jetzt schon beinahe Courage dazu, gegen die Sezession zu sein. Sie ist eine Mode geworden, mit der ganzen unsinnige Tyrannei, die Mode haben." En alemán original, traducido por el autor.
} 


\section{La mirada de Adolf Loos sobre el café vienés como tercer lugar}

implementar las ideas sezessionistas recogidas en el Ver Sacrum ${ }^{12}$ sobre sus estudiantes, conduciéndoles eventualmente a la Werkstätte, la cual contaría con un programa de enseñanza puesto en práctica en 1905 (Jensen 1996), año de disolución de Sezession y momento en que los talleres que conformaban la Wiener Werkstätte toman automáticamente su relevo (Fiell and Fiell 1999).

La Wiener Werkstätte evoluciona desde la Sezession con un punto de partida similar; la voluntad de crear el trabajo de arte total, Gesamtkunstwerk. Los asociados a la Wiener Werkstätte ${ }^{13}$ retan a la sociedad industrializada mediante la realización de manufacturadas desde una gran diversidad de campos, compartiendo un mismo paraguas estilístico que conformaba un estilo unificado simple y práctico (Fahr-Becker 2008). La Wiener Werkstätte consigue armonizar los motivos geométricos con sutiles formas orgánicas o naturales (Fahr-Becker 2008), aún compartiendo características formales con la Sezession. Sin embargo, la Wiener Werkstätte pone más atención sobre el trabajo artesanal realizado por expertos tradicionales (Fahr-Becker 2008), trabajando con metal, con piedra, con madera, con piel, y experimentando con materiales como el oro, metales, piedras preciosas o papel maché en un estilo caracterizado por la geometría, la simetría, y las formas simples, dejando huella posteriormente sobre el Art Decó y la Bauhaus (Fahr-Becker 2008) (Figura 1).

El nacimiento mediático de la Werkstätte, y sus posteriores apariciones constantes en la prensa se ganan rápidamente la popularidad en opinión pública (Hevesi 1906). La estrategia de impacto sobre el tejido socio-cultural desde el la perspectiva discursivo-narrativa ideada por Zuckerkandl no sólo considera en el lanzamiento a la popularidad de la Werkstätte por medio de la prensa escrita, sino que también lo hace desde la difusión oral en los espacios de discusión ${ }^{14}$ intelectual. El café Vienés es donde la idea del nacimiento de la Sezession se gesta, así como posteriormente, la decisión de apoyar a la Werkstätte desde la crítica y con consecuencia intencionada de impacto a la opinión pública (Hevesi 1908) ${ }^{15}$.

En este contexto de nacimiento de tendencias estilísticas y de diseño con una componente intelectual imperativa, al otro extremo de la narrativa de la Werkstätte se encuentran otra nueva generación de intelectuales incluyendo a Dagobert Peche, Josef Frank, Oskar Wlach y Oskar Strnad, los cuales critican el concepto de

\footnotetext{
12 Revista de difusión de obras y pensamiento publicada por la Sezession vienesa entre 1898 y 1903. ${ }^{13}$ El propio nombre, Wiener Werkstätte, es un indicativo de su ideal. El conjunto de la Wiener Werkstätte lo constituyen diversos talleres o workshops por lo que se habrían llamado Wiener Werkstätten en plural. Sin embargo, la denominación Werkstätte responde a la intencionalidad de manifestar el ideal de Gesamtkunstwerk o obra de arte total; el conjunto de talleres, o los Wiener Werkstätten eran en sí mismos una Gesamtkunstwerk, un solo taller, una sola obra de arte total que integraba todas las artes (Fahr-Becker 2008).

14 Discusión o Lectura Pública: Se utilizan como equivalentes a los términos en inglés public speaking y public discussion. Se caracteriza por la acción oratoria pública para informar, influenciar o entretener a una audiencia determinada.

15 Hevesi repite esta historia en el prólogo que escribe para la antología de Zuckerkandl Zeitkunst. Nicholas Powell describe la historia en The Sacred Spring: The Arts in Vienna, 1898-1918. Greenwich, Conn.: New York Graphic Society, 1974. P. 117. También citado por Hess Fiell and Fiell.
} 
Gesamtkunstwerk inclinándose por la emancipación de la utilidad, llegando a expresiones artísticas y estéticas con la utilidad o funcionalidad relegada a un segundo plano (Witt-Dörring 2013b). Ellos se inspiran en figuras naturales orgánicas y más propias del Art Noveau (Fahr-Becker 2008), influidas por la etapa historicista de las décadas anteriores, bebiendo del rococó o el barroco (Fiell and Fiell 1999).

\begin{abstract}
"Wir wollen einen innigen Kontakt zwischen Publikum, Entwerfer und Handwerker herstellen und gutes, einfaches Hausgerät schaffen. Wir gehen von Zweck aus, die Gebrauchsfähigkeit ist uns erste Bedingung, unsere Stärke soll in guten Verhältnissen und in guter Materialbehandlung liegen" 16.

Buscamos crear un contacto íntimo entre el público, el diseñador y el artesano, para crear objetos buenos y simples. Partimos del propósito de que la utilidad es la prioridad más alta, y que nuestra fuerza debe recaer en las buenas proporciones y el buen tratamiento de los materiales.
\end{abstract}

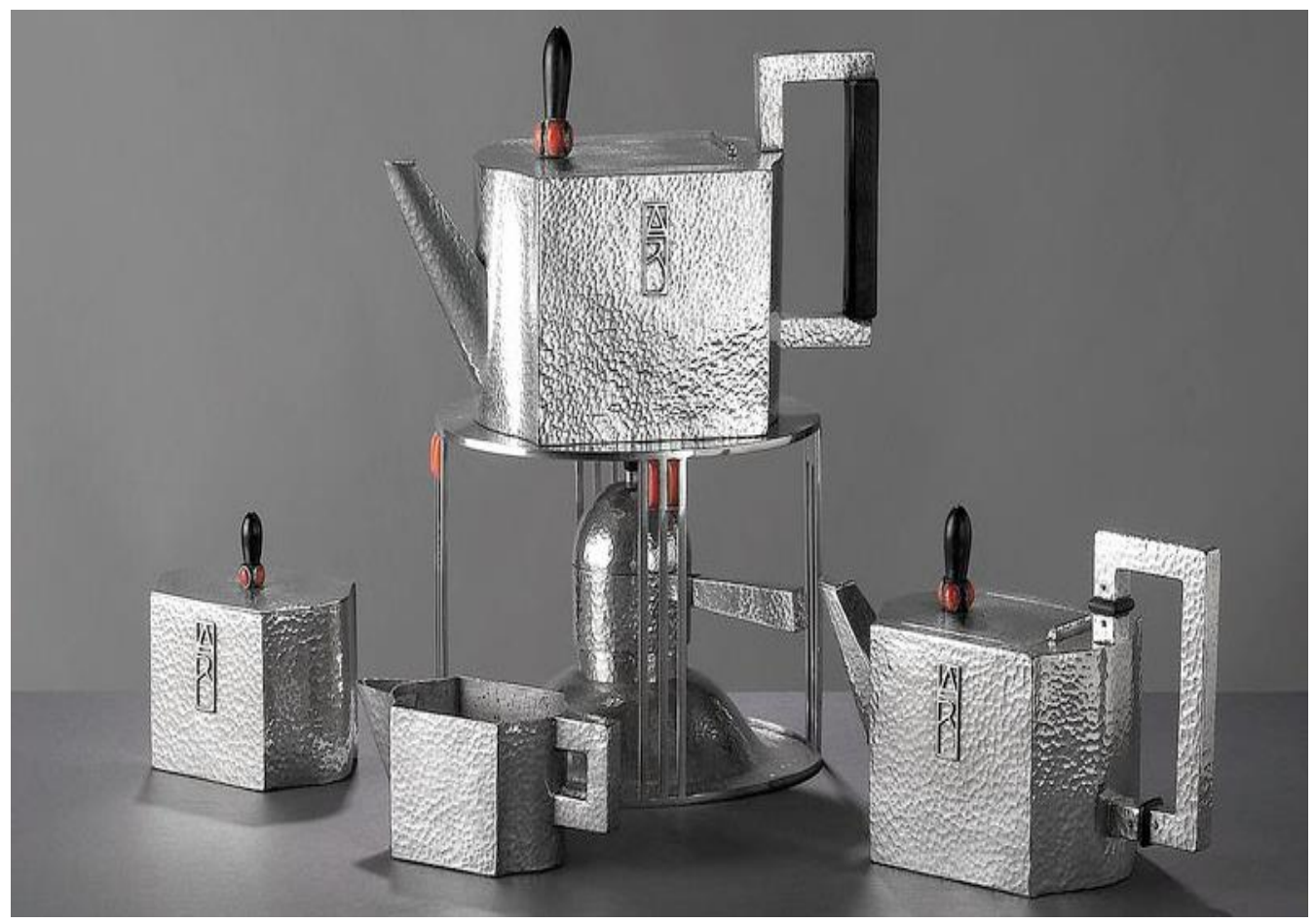

Fig. 1. Imagen de un juego de té diseñado por Josef Hoffmann. Manufacturado por Konrad Koch para la Wiener Werkstätte en 1903. Imagen realizada por Georg Mayer (C) MAK Museum Viena.

Der Jugendstil toma el nombre de la revista Jugend publicada por primera vez en Múnich en 1896 (Fiell and Fiell 1999). Este grupo de artistas no pretende

\footnotetext{
${ }^{16}$ Fragmento del manifiesto en el que se basaba el programa de estudios de la Wiener Werkstätte, basado en los pensamientos generales de Ver Sacrum y el estilo utilitarista de Otto Wagner escrito por Hoffmann, J. y Moser, K. En alemán original, traducido por el autor.
} 


\section{La mirada de Adolf Loos sobre el café vienés como tercer lugar}

únicamente una reforma del arte historicista, sino que se inclina hacia un estilo de vida independiente de las tendencias comerciales, siendo esta la crítica principal hacia los sezessionistas, estudiando la naturaleza desde un punto de vista científico y sirviendo de inspiración para sus diseños caracterizados por el dinamismo y el desarrollo orgánico, apostando por motivos vegetales manieristas apoyados por estudios morfológicos de patrones de crecimiento desde la botánica (Fiell and Fiell 1999, Fahr-Becker 2008).

El pensamiento y las ideas de Gesamtkunstwerk - el trabajo de arte total compartidas por los sezessionistas y los miembros de la Wiener Werkstätte se sitúan diametralmente opuestos a Adolf Loos y su alternative Weg in die Moderne, camino alternativo hacia la modernidad (Witt-Dörring 2013a). En el marco de estas tendencias, Adolf Loos recibe una cierta influencia en sus primeros años en Viena de Karl Mayreder, profesor de la Technische Hochschule quien, abierto a nuevas ideas, le transmite su interés por los problemas de índole urbano y el lenguaje de la antigüedad clásica (Carranza Macías 2016). En consecuencia, Loos se posicionaba en una modernidad cultural, en contraste con la modernidad formal y estilística de sus coetáneos (Witt-Dörring 2013b). Para Loos, la modernidad es una cuestión que se acerca más a la actitud que a la intención de desarrollar un estilo determinado basado en la ornamentación asociado a los sezessionistas y caracterizando al Jugendstil. Loos habla de que la unidad de arte y funcionalidad en los objetos utilitarios era un proceso carente de interés (Witt-Dörring 2013b). Respeta a Otto Wagner, al considerarlo el único sezessionista capaz de diseñar teniendo en cuenta tanto el arte como la funcionalidad, es decir, sin sobreponer su propia expresión artística sobre el trabajo tradicional de los artesanos y con el fin de crear un belleza artificial que estuviera presente en la vida de los objetos, intentando modificar la vida de las personas y el uso que éstas hacen de aquéllos. Los diseños de Loos no responden exclusivamente a una voluntad de estilo específica - en el sentido de la Kunstwollen riegeliana -, sino que tanto el estilo como función - e.g. la distribución programática de las viviendas según su concepto de Raumplan en el que cada espacio es dotado de las dimensiones que el propio espacio necesita - son consecuencia del estudio de los hábitos y las necesidades del cliente concreto, y de la respuesta que se da a sus necesidades específicas. Él estudia el descanso y el espacio personal (Arnau Amo and Gutiérrez Mozo 2014). En sus escritos valora significativamente las superficies textiles, alfombras, tapices, cortinas, y se inclina por las telas como representación de la calidez, de la intimidad ${ }^{17}$.

\footnotetext{
${ }_{17}$ Un ejemplo es la habitación de Lina y Adolf Loos en Bösendorfrestraße, Viena, donde prácticamente la totalidad del espacio está cubierto de textiles. La suavidad y calidez que transmiten y representan corroboran la percepción de los interiores como feminidad, erotismo e intimidad. También es evidente en Loos la influencia anglosajona tanto inglesa como americana observando, en el caso de los interiores, no únicamente con repetidas referencias al mobiliario de Hepplewhite y Chippendale en la forma de las curvas y los materiales, sino a la presencia explícita de las mismas en algunos de sus interiores.
} 


\section{Jesús López Baeza}

\section{El papel del espacio social}

Cuando Loos resulta conocido como arquitecto, participa en una primera serie de artículos para la Neue Freie Presse. Fue por el aniversario del reinado del Kaiser Franz Josephs en 1898 en la Imperial Jubilee Exhibition, cuando tiene lugar una exhibición en el Prater de Viena sobre objetos de diseño austriacos. Esta exhibición trata de mostrar el público el nuevo estilo de Austria, con la intención de exponer el carácter metropolitano de Viena al resto de Europa para reafirmarse como capital cultural. En este contexto, tienen lugar múltiples conferencias, lecturas y debates y "la nueva ciudad moderna se construye a través del discurso" (Witt-Dörring 2013b). En este foro, Loos aprovecha la oportunidad para discutir y poner de manifiesto sus discrepancias sobre aquellos productos y arremeter contra los sezessionistas. Su estilo ingenioso y la elevada crítica a los estilos austriacos lo convierten de la noche a la mañana en el portavoz de una generación que pretendía acabar con el siglo XIX (Stuiber 2012).

El proceso de transmisión de conocimiento en lecturas públicas es complejo, y es estudiado con la intención de obtener un determinado resultado para situarse en el espacio social de una ciudad. Stewart (2009) explica la afirmación de Ludwig Wittgenstein en la que enuncia que los límites del contexto espacial y temporal son fluidos. Wittgenstein introduce el concepto de juego de lenguaje como una interpretación particular del mundo, en la que el objeto de estudio es el discurso que se mueve entre el contexto pragmático del discurso propio y la situación espaciotemporal del momento en el que el discurso se produce (Stewart 2009). La personalidad del orador es un elemento fundamental, ya que no es únicamente relevante lo que se dice, sino también quién lo dice, cómo lo dice y a quién lo dice. Es así como lo que se pone en juego es la performance, no únicamente el discurso textual (Stewart 2009). Por ello, el personaje de Adolf Loos no puede ser comprendido, en la época actual, únicamente desde sus escritos (Arnau Amo and Gutiérrez Mozo 2014).

Heather Hess describe a Loos como el mayor rival ideológico de la Wiener Werkstätte (Hess 2006). Antes de 1903, los trabajos tanto de Loos como de Hoffmann son enormemente mediáticos y llenan páginas de periódicos y revistas. No obstante, después de esa fecha, Loos desaparece de la prensa arquitectónica escrita. Continúa su cruzada dialéctica contra la Werkstätte mediante sus propios escritos y lecturas públicas. Según Colomina (1994), Loos era un personaje que no se llevaba bien con la prensa arquitectónica. Sus obras no son para ser fotografiadas (Arnau Amo and Gutiérrez Mozo 2014), imposibilitando así su difusión y, por tanto, que éstas se comprendan correctamente desde sus narrativas espaciales e intelectuales. Él mismo explica que cada espacio y cada pieza de mobiliario están diseñados para las necesidades específicas el cliente y su modo de habitar. El intento de transmitir las piezas, difundirlas y reproducirlas sería fútil, ya que tanto su utilidad como su entendimiento no estarían enfocadas al lector (Loos 2012b). " La arquitectura fotogénica es mala arquitectura”(Loos 2012c) y se ejemplifica con su 


\section{La mirada de Adolf Loos sobre el café vienés como tercer lugar}

diseño del Café Museum, donde la correcta representación de éste no radica en las fotografías que fueran difundidas por las revistas, sino en la experiencia propia de su interior (Loos 2012a).

Nos encontramos, así, con un personaje de confrontación y con una controversia potencial. Schwartz (2012) investiga la persona de Adolf Loos y sus relaciones puntuales con lo que él llama el caso; "una serie de prácticas que involucran la convergencia de investigaciones científicas, procesos legales, y la esfera pública”18. Los encuentros de Loos con el caso son de una controversia particular, y están siempre relacionados con la intersección entre el reino de lo público y lo privado (Schwartz 2012). De este modo, es inevitable considerar que estos encuentros influyeran en su propia concepción de este límite, y consecuentemente, en su arquitectura y su manera de diseñar espacios.

Uno de sus encuentros con el caso tiene lugar en 1910, con una discusión respecto a la fachada del edificio para la sastrería Goldman und Salatsch (Schwartz 2012) actualmente conocida como Looshaus en Michaelerplatz -. Aquí la fachada del edificio se considera inapropiada estéticamente y en consecuencia es llevada a discusión pública. Loos defiende su diseño ante el público en discusiones, las cuales son consideradas un elemento de promoción de su arquitectura y su pensamiento más que la propia arquitectura sobre la que se discute. Afirma Schwartz que la decisión final - colocar maceteros decorativos en los alféizares - es producto de una negociación pública y lo propone como ejemplo de cómo el público - o el espacio social - influye directamente en el espacio físico. Afirma que "planeado o no, la fachada del edificio Goldman und Salatsch es como es, porque lleva las huellas de esta negociación" (Schwartz 2012). Colomina (1994) cuestiona las causas y las intenciones de este diseño y esta polémica, planteando que quizás fuera una fachada inapropiada inicialmente, con la intención de generar discusión, y así defender y difundir públicamente su concepción de la arquitectura, proponiendo que la figura de Adolf Loos es controversial de forma intencionada, con la finalidad de suplir la ausencia en la prensa arquitectónica escrita. En este sentido, Loos provoca polémica desde la sátira como una estrategia publicitaria para hacer llegar su arquitectura al reino de lo público, apoyándose en su posición social y en su amistad con el periodista Karl Kraus (Schwartz 2012).

La arquitectura residencial de Loos es una arquitectura de contrastes que diferencia lo privado de lo público con una máscara plana y aséptica que oculta y protege la intimidad del verdadero acto de habitar. Dos de los encuentros de Loos con el caso se caracterizan por un factor fundamental: se hace público algo que originalmente es privado ${ }^{19}$. Sus diversas apariciones en prensa y discusiones públicas y sus tácticas publicitarias ponen de manifiesto algo que su arquitectura transmite de forma

\footnotetext{
${ }^{18}$ En inglés original, traducido por el autor.

${ }^{19}$ En 1905 participa como testigo en el caso de Theodor Beer, y en 1928 es acusado. En ambos casos, las acusaciones se centran en la posesión de fotografías de carácter privado de personas menores de edad, centrándose la discusión en la naturaleza artística o delictiva de dichas fotografías y con gran impacto mediático. (Schwarz 2012).
} 
inequívoca: Él insiste en una separación radical entre el interior y el exterior como un modo de preservar el espacio para la intimidad y el habitar. El espacio interior debe ser subjetivo, propio y adaptado a la persona que lo habita. El exterior, la fachada, ha de ser muda, ha de ser una máscara que proteja al ocupante, sus deseos y sus necesidades más vitales. La diferencia entre el interior y el exterior es producto de la idea de censurar la intimidad, de recoger y guardar lo que es propio y proyectar hacia el exterior un carácter diferente frente, una personalidad social distinta de cara al público (Colomina 1992). La arquitectura de Adolf Loos es una arquitectura de contrastes y separación. Separación entre interior y exterior, entre público y privado, entre hombres y mujeres, entre invitados y anfitriones, y entre los miembros de la familia y el servicio. De manera análoga, la estética del exterior está separada del interior. Una es simple, plana y geométrica, y la otra cuenta con una enorme variedad de superficies, colores, texturas y materiales.

"El exterior debe asemejarse a un traje de etiqueta, una máscara masculina, como ser unificado y protegido por una fachada anodina. El interior es la escena de la sexualidad y la reproducción, y de todas las cosas que separan al sujeto del mundo externo" (Colomina 1992) ${ }^{20}$.

El trabajo de Loos está enfocado a la acción de habitar, con la interacción de los habitantes con el espacio que los rodea, donde el individuo y el espacio se transforman uno a otro en un proceso de apropiación ${ }^{21}$ (Heynen and Baydar 2005). Es por ello que Loos arremete los estilos intencionados con la voluntad de convertirse en arte, de Kunstwollen riegeliana ${ }^{22}$. Él se sitúa bajo el paraguas se Gotfried Semper, supeditando el estilo estético a las artes técnicas y tectónicas fundadas en los materiales y en las herramientas que les confieren forma y utilidad (Arnau Amo and Gutiérrez Mozo 2014). Loos confía en los materiales para los revestimientos y acabados, ya que son los que contactan directamente con la vida que habita el interior de la arquitectura. La casa no es más que la estructura que sustenta estos materiales (Arnau Amo and Gutiérrez Mozo 2014; Loos 2012b).

\section{El café como lugar}

Las lecturas públicas son efímeras, son eventos temporales que tienen lugar durante un periodo de tiempo limitado. Esta tenue durabilidad causa una tensión que contribuye al atractivo de las mismas (Stewart 2009). Una lectura pública transforma un espacio en un lugar, desde el punto de vista de la teoría espacial de

\footnotetext{
${ }^{20}$ En ingles original. Traducción de A.M.R.

21 "Appropriation"en inglés original, traducido por el autor.

22 Sobre la Kunstwollen, Riegl escribe: "Hay algo en el hombre que le lleva a encontrar placer en la belleza formal, algo que ni nosotros ni los seguidores de la escuela que explica el origen de las artes por la técnica y los materiales somos capaces de definir" Citado por Arnau Amo y Gutiérrez Mozo (2002).
} 


\section{La mirada de Adolf Loos sobre el café vienés como tercer lugar}

Duarte (2007) la cual establece como elemento constituyente del lugar la componente social intangible, frente al espacio como el substrato en la que ésta sucede. Desde esta perspectiva, Stewart (2013) realiza una taxonomía de los lugares donde las lecturas públicas se desarrollan. Las analiza en términos de movilidad, extensión y localización, y los categoriza según el grado de carácter público o privado, siendo el más público la calle o la plaza, y el más privado el salón de una casa. El café se encuentra en el estadio central, que denomina Dissemination and Dialogue, Diseminación y Diálogo. Stewart (2009) se refiere a Hans Schliepmann al explicar que aunque los cafés no fueran específicamente diseñados para la divulgación, influyeron imperativamente en la historia como espacios de comunicación (Stewart 2009). El café, en toda su complejidad y riqueza, es un supremo protagonista en la historia intelectual de Europa y, en particular, de la ciudad de Viena.

"La fructuosa relación entre la actividad intelectual y el café ha creado una dimensión pública emblemática más allá del espacio en sí" (Pireddu, 2013).

El café se asocia de igual manera al espacio físico como al espacio cultural intangible al que Rittner, Haine y Jackson bautizan como The Thinking Space (Pireddu 2016). En este sentido, tanto la capital francesa como la austriaca han demostrado que el interior de los cafés son un catalítico para el desarrollo de movimientos filosóficos y de pensamiento crítico, y son asociados por varios estudios realizados por Haine (1998) con nombres de intelectuales y escritores particulares (Pireddu 2016).

El café es considerado dentro del marco conceptual de la teoría espacial, como un tercer lugar que se sitúa en la frontera entre la esfera de lo público y la del espacio doméstico privado con multitud de elementos en común con el ambiente doméstico, el confort, y las dinámicas sociales y de comportamiento, lo sitúan precisamente en esta intersección. Pireddu (2013) afirma que la propia consciencia de la modernidad urbana del cambio de siglo es expresada por Baudelaire desde una silla en un café icónico de Montmatre que también vio nacer al Dadaísmo y el Surrealismo (Pireddu 2013). La cultura de la cafetería vienesa fue importada de los modelos parisinos y adaptada a su propio contexto, convirtiéndose en un foro intelectual y cultural para reuniones de la aristocracia, la burguesía, artistas e intelectuales (Timms 2013), también considerado una extensión del salón propio de una casa Kurdiovsky (2013) Carranza Macías (2016) explica que en la planta baja de la Griensteidl-Haus en Michaelerplatz (Figura 2) se ubicaba el café homónimo que, en 1890 ve nacer en su interior al movimiento literario de la Jung-Wien, caracterizado por la voluntad de ruptura con la generación previa de intelectuales burgueses y su síntesis historicista. 


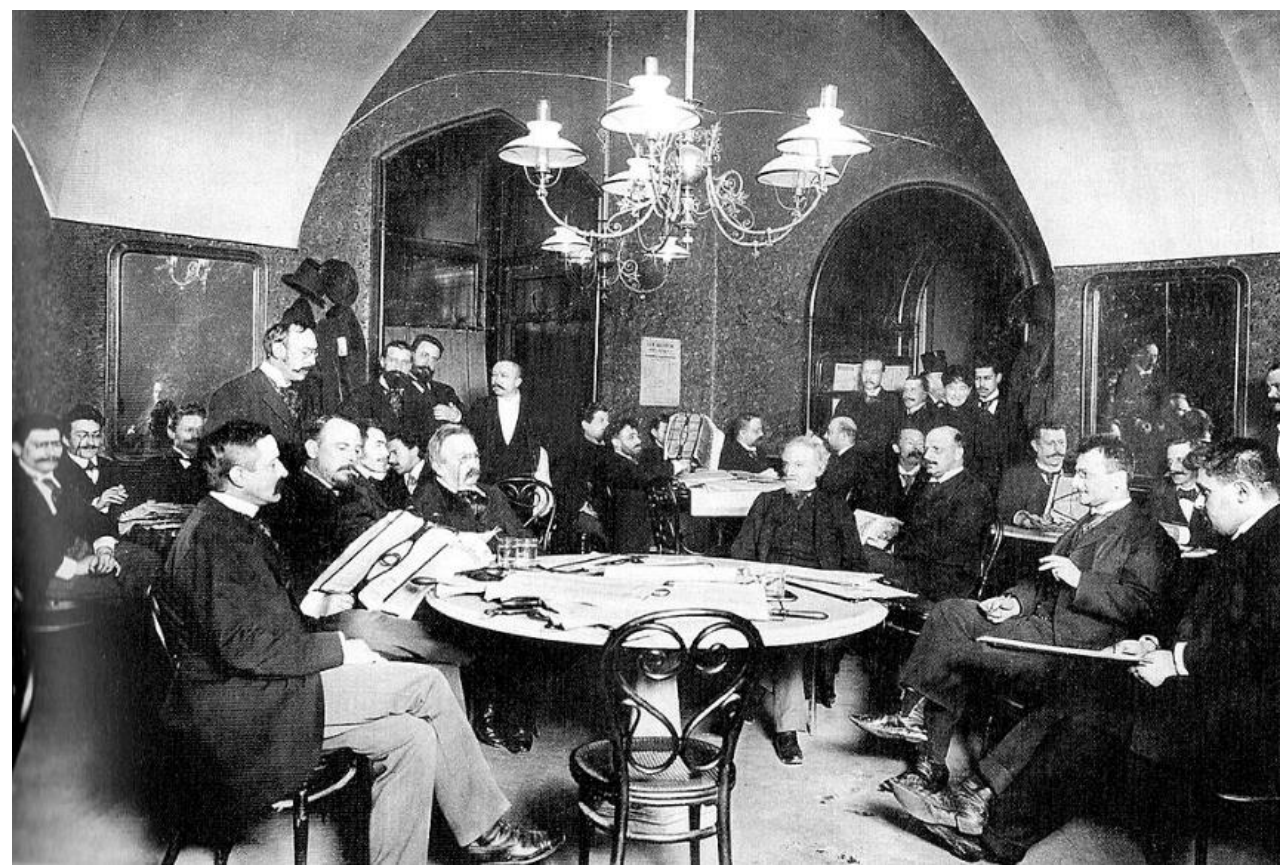

Fig. 2. Imagen del interior del Café Griensteidl en 1897. Tomada por Carl von Zamboni. (C) Wien Museum. 2006.

Es muy probable que fuera en este café, en Griensteidl, antes de su demolición, donde Adolf Loos comenzara a relacionarse con los círculos intelectuales y artísticos más elevados (Carranza Macías 2016). Fue aquí donde conoció a su amigo y periodista Karl Kraus, escritor de la Jung-Wien, quien le transmitió las ideas de las nuevas formas de prensa y opinión pública, de la nueva crítica y la difusión de ideas, desde donde concluiría que la prensa influye de manera determinante sobre el espacio social. De esta manera, la publicidad y la privacidad, lo que muestran, lo que transmiten y lo que ocultan para la intimidad, son conceptos que Loos incorpora a su concepción de la arquitectura y el acto de habitar. También en el interior de este café, ambos Adolf Loos y Karl Kraus conocen a Peter Altemberg, que "ejercía un fuerte magnetismo entre los jóvenes escritores y artistas de la ciudad" (Carranza Macías 2016). Con la demolición del café Griensteidl en 1897, los artistas e intelectuales que con frecuencia se reunían allí se trasladaron al Café Central (Carranza Macías 2016), situado en Herrengasse a pocos metros de Michaelerplatz. Es también en una de estas cafeterías donde nace el grupo que dará lugar en 1897 a Sezession (Fahr-Becker 2008); en el café Sperl, situado en Gumperdorfer Straße, a dos calles de distancia del lugar donde se erigiría su sede un año después, diseñada por Joseph Maria Olbrich. A su vez, este edificio se encontraría en Operngasse, calle en la que en 1899 abriría sus puertas el conocido Café Museum (Fig. 2) de Adolf Loos. Seis años después de la Sezession, la Wiener Werkstätte se gesta también en una discusión de cafetería (Fahr-Becker 2008). 


\section{La mirada de Adolf Loos sobre el café vienés como tercer lugar}

\section{El café según Loos}

Es evidente el estrecho vínculo que une el café vienés con la sociedad artística e intelectual de la época. El café Museum, diseñado por Adolf Loos e inaugurado en 1899, es conocido por ser el encuentro tradicional de intelectuales como Karl Kraus y Peter Altembeg en el campo de la literatura, Egon Schiele, Oskar Kokoschka y Gustav Schütt en la pintura, Alan Berg y Oscar Straus en el campo de la música, o el caricaturista y cantante de cabaret Carl Hollitzer, quien lo consideraba como una segunda casa (Segel 1993). Esta última afirmación pone de manifiesto el carácter de tercer lugar del café - definido por el mimo Oldemburg (1989) como una casa lejos de casa, a home away from home - el cual ofrece características domésticas de privacidad al mismo tiempo en que éstas intersectan con la interacción social en público. Los terceros lugares se definen por características como la conversación como elemento central, la asiduidad de los asistentes, la neutralidad del espacio, el confort y y el ambiente alegre o playful (Oldemburg 1989).

El café Museum se sitúa en la planta baja del edifico que se sitúa entre Operngasse y Karlplatz, a escasos metros del edifico de Sezession. Debido a su condición de esquina, la planta del edificio dibuja una forma de "L" y está dividida en tres espacios diferenciados: una sala en el brazo más largo, el hall de entrada en el vértice, y una sala en el brazo más corto. Está probablemente inspirado por el Romantisches Café diseñado por Franz Schwechten en Berlín, con una planta similar, y donde también arcos definen en espacio interior y delimitan las salas (Stewart 2009). De una forma similar al Romantisches Café, en el Café Museum la sala de menor tamaño era llamada la pool of swimmers o piscina de los nadadores y estaba reservada para las personas que se habían ganado su nombre y con él, un asiento en la sala y un puesto de relevancia en el mundo intelectual (Stewart 2009). La sala mayor, sin embargo, era frecuentada por jóvenes con aspiraciones intelectuales y culturales que esperaban llamar la atención de alguno de los presentes en la poolo piscina (Stewart $2009)^{23}$. En la sala mayor se encuentran dispuestas en fila tres mesas de billar o pool tables. En este caso concreto, no es la arquitectura en sí misma la principal cualidad del espacio interior del café, sino que se convierte en un contenedor de que integra una colección de actividades, dinámicas y relaciones sociales que dan lugar a la definición de este ambiente interior. En otras palabras, la protagonista del café Museum no es la arquitectura de éste, sino el conjunto de relaciones que suceden en ella, de manera que la concepción de la arquitectura como estructura que sostiene al acto de habitar es aquí extrapolada a la escena pública y a un espacio que no se habita, sino que vive. En este caso y desde la perspectiva de Duarte (2007), las salas del Café Museum se considerarían espacios como substrato del lugarque constituye el ambiente social intangible presente en dichos espacios. En línea con esta idea de espacio neutro conteniendo el gran valor intangible social y discursivo, el

\footnotetext{
23 En inglés original, traducido por el autor. También puede considerarse el término pecera, como refiriéndose a que en ella nadan los peces gordos.
} 
sezessionista y crítico de arte Ludwig Hevesi lo bautiza como Café Nihilism por su característica carencia de ornamentación y su simplicidad estilística (Segel 1993). Hevesi (1908) afirma que el estilo de Loos se hace evidente en este café en su distancia al sezessionismo. Sin embargo, no lo considera un enemigo de la Sezession, sino que lo de lo describe como una visión complementaria.

En el vértice se encuentra el hallde entrada (Fig. 3), con un elegante recibidor frente a la puerta sobre el que se exhiben pasteles y dulces. Este expositor puede percibirse desde el exterior, mirando a través de la puerta, y es el elemento que da la bienvenida a quien entra en el café. La gran variedad de dulces se exhiben de una manera sensual y sugerente y son multiplicados por los espejos que se sitúan tras ellos. Además, es en el trayecto hacia ellos, desde la puerta hacia el expositor, cuando la persona que acaba de entrar al café percibe de manera simultánea el ambiente de las dos salas, y análogamente es vista por las personas de ambas salas, siendo cada sala no es visible desde la otra, pero debido a la condición de la planta en forma de esquina.

La fachada del café está revestida de un mortero color crema plano y uniforme. Con un primer acercamiento, podría afirmarse que lo que se pretende con una fachada de estas características es similar a lo que persigue Loos con las fachadas en su arquitectura residencial: ocultar y proteger la intimidad de los interiores. Sin embargo, en el café Museum se ponen en juego las ventanas como un elemento fundamental en la relación entre interior y exterior. Se diseña un set de dos cortinas por cada ventana, situándose a diferente altura y profundidad en el hueco. La cortina superior es encargada de controlar la entrada de luz a la sala y se encuentra abierta. Por el día contribuye a la sensación de amplitud y por la noche irradia la luz del interior hacia la calle, convirtiendo el espacio interior en un escaparate de sí mismo. No obstante, una segunda cortina está situada en la parte inferior de los huecos, a la altura de los ojos de quien está sentado en la mesa situada frente a las ventanas. Ésta es la encargada de la intimidad, ya que puede ser abierta o cerrada a voluntad del cliente. Así, iluminación e intimidad quedan desvinculadas.

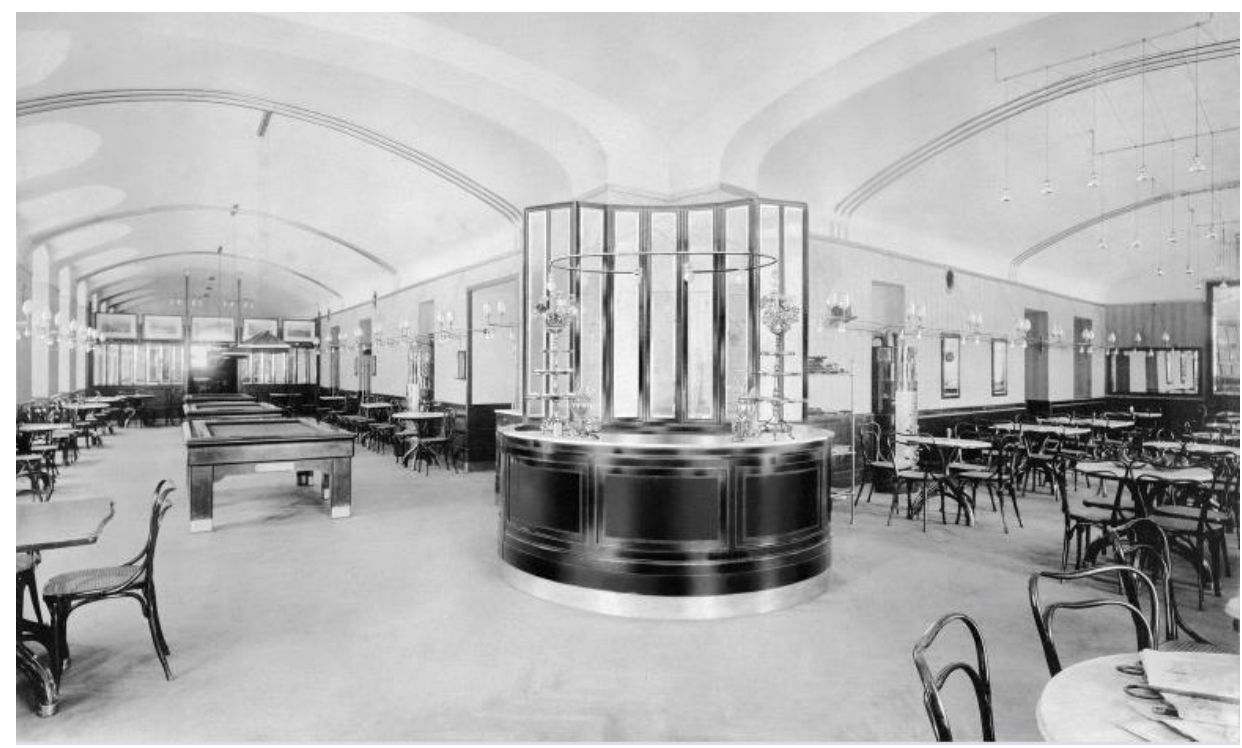

Fig. 3. Imagen del interior del café Museum desde el hall de entrada. (C) Albertina Museum. 
El café es un lugar público y privado de manera sincrónica: el cliente pasa en ellos un lapso de tiempo relativamente largo, pero no es dueño del espacio ni de los objetos que se encuentran allí. Debe compartir el espacio y formar parte del ambiente sin ser sujeto activo de modificaciones, sino de recibir un determinado diseño espacial y adaptarse a las condiciones de éste para utilizarlo de una manera prolongada en el tiempo $\mathrm{y}$, con gran probabilidad, reiterada. Gracias a esta recurrencia el cliente reconoce lo objetos, los espacios, y la dimensión social de los mismos. Reconoce los agentes que intervienen y las relaciones y procesos que tienen lugar entre éstos, a otros clientes, al servicio, horarios, eventos, quién se sienta habitualmente en cada mesa, etc. Así, el café se convierte en un tercer lugarinterior, público, y cuyas condiciones físicas y sociales permanecen constantes. Esta invariabilidad permite al cliente anticiparse y reconocer elementos y procesos y, por tanto, aumentar la sensación de conocimiento y control de los mismos. Este fenómeno se traduce en un incremento de la sensación de confort que tiene como consecuencia que la asiduidad se mantenga. Cuanto más se frecuenta el café, mejor se conoce y, por tanto, más se frecuenta.

De esta manera el conocimiento, por medio de la capacidad de anticipación, está vinculado a la sensación de control sobre la situación. Es por este motivo por el que los cafés de mayor tamaño tienden a simular una reducción de escala, ya que una menor cantidad de información para asimilar supone un incremento de la capacidad de anticipación y, por tanto, de la sensación de control ligada al confort. Un espacio menor contiene menos cosas. Por lo tanto, la sensación de un espacio de menor tamaño es más confortable que un gran espacio en el que el individuo está expuesto. La reducción de escala se pretende, en casos como el café Sperl, el café Prückel, o el mismo café Museum, con la colocación de revestimientos verticales hasta una altura límite, diferenciando la parte superior de las paredes. También, la altura de las lámparas y los apliques se controla, siempre a una cota determinada. Dejando la parte superior de los muros, y los techos sin revestir ni iluminar, la percepción de los mismos se reduce y la escala se acota, incrementando el confort.

Las relaciones de visibilidad y exposición de las personas que se encuentran en el interior de los espacios con respecto a las que se encuentran en el exterior, o las que acaban de entrar, es un tema recurrente en la arquitectura de Adolf Loos en este tipo de terceros lugares. También sucede con las situaciones de control, o jerarquización de los agentes. En múltiples ocasiones, la arquitectura residencial de Loos sitúa unos espacios por encima de otros, prestando atención a las iluminaciones y los contrastes favoreciendo la situación de control de unos agentes sobre otros. Intimidad y control son dos aspectos fundamentales en la arquitectura residencial de Loos (Colomina 1992).

Loos diseña el American Bar en 1908 con una ilusión de amplitud situando espejos en la parte superior de los cerramientos - cercanos al techo - que no son accesibles desde la perspectiva visual de los clientes, de manera que no compromete la intimidad de los mismos (Fig. 4). Estos espejos multiplican el espacio mediante el 
efecto óptico de extender la dimensión de los techos, los cuales parecen continuar aunque la vista no acceda a ver el final. Estos espejos reflejan únicamente espacios, no personas. La situación de control también se consigue mediante la posición de los asientos respecto a las mesas: todos los bancos se encuentran de espaldas a la pared, permitiendo a los comensales observar lo que sucede en el espacio central de bar.

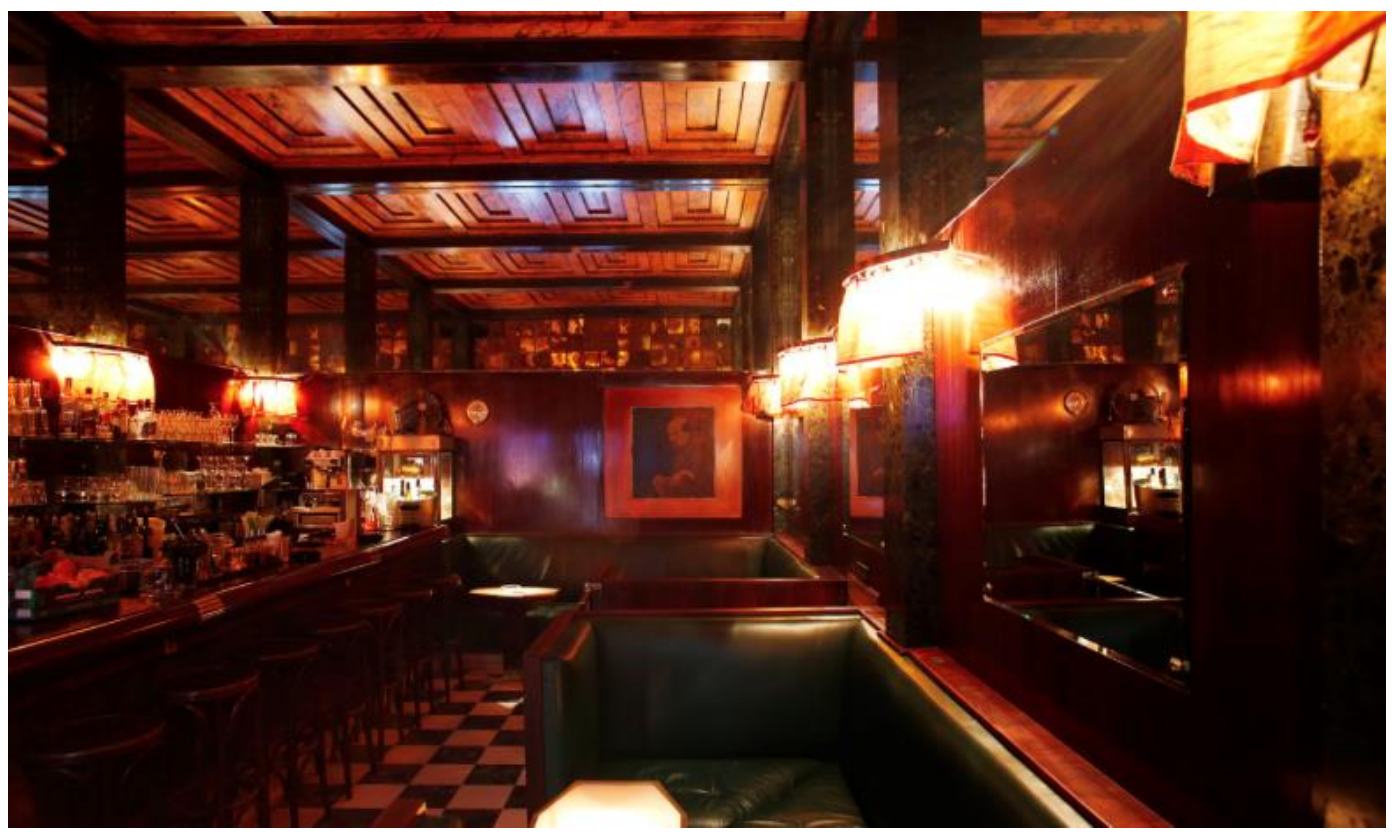

Fig. 4. Imagen del interior del American Bar en la actualidad, tras su reconstrucción a su estado original. (C) Albertina Museum.

De manera similar al café Museum, la relación con la calle también es objeto de atención. Sin embargo, aquí no se pretende mostrar el interior como un escaparate, sino que se oculta del exterior. La fachada la componen tres puertas. La central, es la de acceso, pero no conecta visualmente con el interior sino con el atrio, en el que se encuentra una segunda puerta de acceso al espacio interior del bar. Las dos puertas laterales dan acceso a dos cabinas individuales con mesas privadas, desde las que los clientes observan y son observados por la calle, pero sin conexión directa con el interior del bar, el cual permanece oculto y protegido de la mirada de los peatones. Además, estas cabinas se encuentran elevadas respecto a la cota de la calle, favoreciendo en cierta medida la situación de dominancia de los clientes respecto a los transeúntes.

Esta preocupación por la visibilidad y las comunicaciones pone de manifiesto que la intención primaria del diseño loosiano de los terceros lugares: el ambiente interior. Lo que realmente define el café Museum es la discusión intelectual que se genera en un interior. Por tanto, con paredes blancas o sin ellas, con ornamento o sin él, la trascendencia del café Museum radica en el juego de relaciones entre espacios, en la exposición y la visibilidad, y cómo ésta se diseña y controla, y en ambiente de carga intelectual que se genera en su interior. Aplicado a la arquitectura 


\section{La mirada de Adolf Loos sobre el café vienés como tercer lugar}

residencial de Loos, el diseño envuelve y protege la tradición. Aquí, es evidente cómo el espacio arquitectónico contiene, paradójicamente, la tradición más profunda y definitoria del ser humano: la comunicación y el diálogo (Colomina 1992).

\section{Conclusiones}

En el momento del cambio del siglo XIX al XX, la ciudad de Viena se reafirma como capital cultural en el centro de Europa. Al igual que en ciudades como París o Berlín, el ambiente del interior de los cafés se convierte en un foro que de alguna manera, tiende a atraer discusiones y debates acerca de arte, cultura y ciencia. Es por ello que las élites culturales de la época se reúnen en ellos con frecuencia.

Es en los interiores de estos cafés donde nace cada una de las corrientes de la modernidad vienesa. Se producen fruto de las discusiones y negociaciones, de las dinámicas sociales que suceden en el interior de estos terceros lugares. Tal y como afirma Florida (2002), unas dinámicas sociales en movimiento constante estimulan la creatividad y el surgimiento de nuevas ideas. Este proceso se traduce en el contacto entre individuos. El tipo de ambiente, la comunicación entre personas pertenecientes a un mismo campo influye directamente en la fluidez en el intercambio de conocimiento entre ellos. Este fenómeno es, según Florida (2002), la clave para comprender el crecimiento, el progreso, y el avance de la sociedad occidental. Desde esta perspectiva, café vienés es un elemento catalizador en la gran zancada que dio la cultura centroeuropea en los alrededores de 1900 y es un ejemplo de cómo papel del espacio social es imperativo para el propio entendimiento de las dinámicas sociales globales asociadas a esta época, conformando un patrimonio cultural en sí mismo.

En este contexto, el pensamiento de Adolf Loos se aleja de sus contemporáneos, inclinándose por el enriquecimiento del modo de vida de las personas que habitan los espacios, desde la comprensión de los mismos modos de habitar. Pone en valor las tradiciones y los tiempos pasados, pero no busca en ellos una referencia estilística directa. Apuesta por una separación radical entre el interior y el exterior en el caso de las viviendas, protegiendo la intimidad de lo que sucede en el interior del espacio mediante una máscara plana, neutra y pura.

En el caso de la arquitectura de los terceros lugares, la atención de Loos no se focaliza sobre la domesticidad, los materiales, o los objetos que representan tradiciones. Sus espacios hablan de relaciones entre personas, situaciones de visibilidad, exposición, o control. Son espacios de característica neutra que permiten y facilitan el desarrollo de la dimensión social, el objeto último de este tipo de arquitectura.

El hecho de que las conexiones visuales, la intimidad, y las situaciones de control sean un elemento definitorio de la arquitectura de Loos, pone de manifiesto cierta correspondencia con su presencia en el caso y su forma de actuar en ellos de cara a su propia concepción de la publicidad, la privacidad, lo íntimo y lo personal. La 
imagen y la opinión pública son elementos determinantes en el desarrollo del caso, y lo que el público conoce y opina son los elementos que establecen el espacio discursivo social. La arquitectura de Loos pone especial énfasis en el diseño de los elementos que regulan la exposición, decidiendo qué es lo que el público puede percibir. Además, autores como Schwartz o Colomina definen al personaje de Loos como una persona mediática, que intencionadamente genera polémica para publicitar sus diseños y su concepción de la arquitectura. Loos controla lo que dice y hace, en definitiva, lo que el público percibe, para generar una imagen pública concreta. Es, por tanto, natural, pensar que su propia arquitectura actúe de la misma manera: su arquitectura está pensada para controlar la percepción desde el punto de vista social, siendo sus vínculos con el caso de probable influencia en (1) la relación de Loos con el público en cuanto a la difusión de su arquitectura y pensamiento mediante la generación intencionada de un espacio discursivo de discusión y (2) su concepción la privacidad y la intimidad, que por tanto, influiría en sus diseños espaciales, tanto de espacios residenciales como de terceros lugares.

\section{Bibliografía}

Arnau Amo, Joaquín, and María Elia Gutiérrez Mozo. "Hacer... Hablar; Hablar... Tal Vez Habitar. Apuntes Sobre Lo Dicho Y Hecho Por Adolf Loos (1870-1933).” I2 Innovación E Investigación En Arquitectura Y Territorio, no. 2:1-24. https://doi.org/10.14198/i2.2014.2.05, 2014.

Bahr, Hermann. "Zur Kritik Der Kritik,” 23-29, n.d.

Bahr, Hermann. “Die Falsche Sezession.” In Secession. Wiener Verlag, 1899.

Carranza Macías, Tomás. "Escaparates de La Modernidad. La Tienda Como Laboratorio de Arquitectura [Dissertation].” ETSAM Madrid, 2016.

Colomina, Beatriz. “The Split Wall: Domestic Vouyerism.” Sexuality and Space 74, 1992.

Colomina, Beatriz. Publicity and Privacy: Modern Architecture as Mass Media. Chicago: MIT Press, 1994.

Colomina, Beatriz. “Adolf Loos. Our Contemporary.” New York: GSAPP, 2013.

Eitelberger, Rudolf Von. "Die Kunstbestrebungen Österreichs.” In Gasammelte Schriften Vol. 2, 1879.

Fahr-Becker, Gabriele. Wiener Werkstätte: 1903-1932. Taschen America LLC, 2008. 


\section{La mirada de Adolf Loos sobre el café vienés como tercer lugar}

Falke, Jakob Von. Geschichte Des Deutschen Kunstgewerbes. Berlin: G. Grote, 1888.

Fiell, Charlotete, and Peter Fiell. Design of the 20th Century. Taschen America LLC, 1999.

Florida, Richard. "The Rise of the Creative Class." The Washington Monthly 34 (5):15-25, 2002.

Folnesics, Josef. Romantik Und Die Kunstgewerbe, 1890.

Franz, Rainald. "Traces of Modernism. Cultural Transfer between Vienna, Bohemia and Moravia 1890-1938." In MAK/GUIDE Vienna 1900, 15-19. Prestel, 2013.

Haine, Scott. The World of the Paris Café: Sociability among the French Working Class, 1789-1914. JHU Press, 1998.

Hess, Heather J. The Wiener Werkstätte and Its Critics. New Brunswick, New Jersey: Graduate School-New Brunswick Rutgers, State University of New Jersey, 2006.

Hevesi, Lajos. Acht Jahre Sezession (März 1897-Juni 1905) Kritik-Polemik-Chronik. C. Konegen, 1906.

Hevesi, Ludwig. "Zum Geleit.” In Zeitkunst Wien 1901-1907, edited by Berta Zuckerkandl. Vienna and Leipzig: Hugo Heller, 1908.

Heynen, Hilde, and Gülsüm Baydar. Negotiating Domesticity: Spatial Productions of Gender in Modern Architecture. Routledge, 2005.

Jensen, Robert. Marketing Modernism in Fin-de-Siècle Europe. Princeton University Press, 1996.

Kurdiovsky, Richard. "The Cliché of the Viennese Café as an Extended Living Room: Formal Parallels and Differences." In The Viennese Cafe and Fin-de-Siecle Culture. Berghahn Books, 2013.

Loos, Adolf. “Architektur." In Ornament Und Verbrechen, 118-69. Vienna: Metroverlag, 2012a.

Loos, Adolf. "Interieurs: Ein Präludium." In Ornament Und Verbrechen, 54-63. Vienna: Metroverlag, 2012b.

Loos, Adolf. “Ornament Und Verbrechen.” In Ornament Und Verbrechen, 94-109. Vienna: Metroverlag, 2012c.

Menz, Christipher. "Vienna: Art \& Design. Klimt, Schiele, Hoffmann, Loos." Melbourne: NGV, 2011. 
Muthesius, Hermann. Wirtschaftsformen Im Kunstgewerbe. Berlin: Verlag von Leonard Simion, 1908.

Pireddu, Nicoletta. "Eds. The Thinking Space. The Café as a Cultural Institution in Paris, Italy and Vienna." Ashgate: Farnham, 2013.

Pireddu, Nicoletta “The Thinking Space. The Café as an Institution in Paris, Italy and Vienna. Ed. by Leona Rittner, W. Scott Haine and Jeffrey Jackson (Farnham and Burlington : Ashgate, 2013).," no. January, 2016.

Oldenburg, R. (1989). The great good place: Café, coffee shops, community centers, beauty parlors, general stores, bars, hangouts, and how they get you through the day. Paragon House Publishers.

Portig, Gustav. Die Nationale Bedeutung Des Kunstgewerbes, 1883.

Reynolds, Diana Graham. Alois Riegl and the Politics of Art History: Intellectual Traditions and Austrian Identity In“ fin-de-Siècle," 1997.

Riegel, Alois. Über Renaissance Der Kunst., 1895.

Schwartz, Frederic J. "Architecture and Crime: Adolf Loos and the Culture of the 'Case."' The Art Bulletin 94 (3):437-57, 2012.

Segel, Harold B. The Vienna Coffeehouse Wits, 1890-1938. Purdue University Press, 1993.

Stewart, Janete. Public Speaking in the City: Debating and Shaping the Urban Experience. Palgrave Macmillan, 2009.

Stewart, Janete. Fashioning Vienna: Adolf Loos's Cultural Criticism. Routledge, 2013.

Stuiber, Peter. "Vorwort." In Ornament Und Verbrechen. Metroverlag, 2012.

Szeps, Berta, and Frau Szeps-Zuckerkadl. My Life and History. Cassell, 1938.

Timms, Edward. "Coffeeehouses and Tea Parties: Conversational Spaces as a Stimulus to Creativity in Sigmund Freud's Vienna and Virginia Woolf's London." In The Viennese Cafe and Fin-de-Siecle Culture. The Viennese cafe and fin-de-siecle culture, 2013.

Varnedoe, Kirk. “. Vienna 1900: Art, Architecture \& Design.” New York: MoMA, 1986.

Witt-Dörring, Christian. "Auf Der Suche Nach Einem Modern Stil." In MAK/GUIDE Vienna 1900. Vienna: Prestel, 2013a. 
La mirada de Adolf Loos sobre el café vienés como tercer lugar

Witt-Dörring, Christian. “Der Wiener Stil.” In MAK/GUIDE Vienna 1900. Vienna: Prestel, 2013b.

Recibido: $12 / 11 / 2019$

Evaluado: 20/12/2019

Versión Final: 02/02/2020 